\title{
Autonomous Base Station Placement for Localization of the GNSS Interference Source
}

\author{
Sun Young $\mathrm{Kim}^{1, *}$, Chang Ho $\mathrm{Kang}^{2}$, and Chan Gook Park $^{3}$ \\ ${ }^{1}$ Mechanical and Aerospace Engineering and Automation and System Research Institute, Seoul National University, Gawnak-Gu, Seoul \\ 08826, Republic of Korea \\ ${ }^{2}$ BK21+ Transformative Training Program for Creative Mechanical and Aerospace Engineers, Seoul National University, Gawnak-Gu, \\ Seoul 08826, Republic of Korea \\ ${ }^{3}$ Mechanical and Aerospace Engineering and the Institute of Advanced Aerospace Technology, Seoul National University, Gawnak-Gu, \\ Seoul 08826, Republic of Korea
}

\begin{abstract}
This paper presents the control strategy of autonomous base station placement for localization of the GNSS interference source. The proposed algorithm deals with the optimization of the base station trajectory for target motion analysis based on bearing only tracking problem. The control strategy of the proposed algorithm is designed to maximize a cost function which is generally a functional of the Fisher information matrix. Compared to the optimal control methods, the proposed algorithm is easy to be designed and implemented, and constraints of multiple base stations' trajectories can be effectively included. In addition, the proposed algorithm also considered target's dynamics that is both uncertain and random, and there are multiple base stations for observing the target. In order to verify the performance of the proposed algorithm, simulation was performed with dynamic target case in the 2D scenario. It is assumed that the base stations' networks have non-fully connected topology. According to the simulation results, it was confirmed that the proposed algorithm presents a flexible control strategy of autonomous multiple base stations' placement for bearing only target tracking system.
\end{abstract}

\section{Introduction}

In recent years, many researches have been conducted to guarantee the quality of satellite navigation system from various interference sources at home and abroad. Especially, importance of the related researches is increasing as South Korea was exposed to the attack of intentional interference source by North Korea several times under special circumstance. It is also necessary to study other types of countermeasures, assuming scenarios where it is impossible to use previous fixed base stations in special situations such as navigation warfare. Therefore, in this paper, we have studied the placements of the mobile stations in order to estimate the position of the interference source even navigation warfare. In this case, only the difference of angle (DOA) measurement is used instead of the differential received signal strength (DRSS) measurement used in the previous study [1,2] in order to estimate the interference's position even in the presence of multiple moving interference sources. The DRSS measurement is difficult to apply for localization in the case of multiple signal sources because it is impossible to judge whether the received signal strength was obtained from which signal source. Using DOA measurements in the presence of multiple sources, both true and false values will be displayed together and the data association process is needed. However, using a filter such as a random finite set filter (RFSF), which is used in the multi- target tracking field, allows tracking of multiple sources without data association. Therefore, in this paper, the DOA measurement is used to estimate the position of a moving interference source using a mobile station, although the accuracy of the position estimation is somewhat lower than using other measurements. In order to improve the accuracy of position estimation, we have studied the placement of mobile stations. The placement performance of base stations was judged using estimation accuracy of the interference source's position and verified by simulation.

\section{Base Station Arrangement}

Studies on the placement of base stations should be considered first in order to precisely estimate the location of GNSS interference signal sources. Especially in an environment where conventional fixed base stations can not be used, as in the navigation warfare situation, it is more important to arrange base stations.

One important role of the base station is to monitor the navigation status and to guarantee the navigation quality by responding to the signal source through the detection and position estimation of the interference signal source.

The GNSS status monitoring is possible on the basis of a single reference station. However, placement studies

* Corresponding author: chanpark@snu.ac.kr 
are more important because more than two reference stations are required for precise position estimation of the signal source and the position estimation performance is influenced by the reference station arrangement.

\subsection{Network Topology}

Arrangement used in the sensor network can be applied for the placement of multiple base stations for the positioning of GNSS interference signal sources. The deployment in the sensor network for location estimation can be largely divided into centralized and distributed (decentralized) deployments.

In general situations with no constraints, centralized deployment has the advantage of higher positional performance and effective location estimation. However, if all of the base stations are not available and only some of the base stations can be used, or if all of the existing fixed base stations become unavailable, such as navigation warfare, additional base station should be located for monitoring the status of GNSS and for estimating the position of the GNSS interference signal source. Thus, in this section, in order to maintain accurate estimation performance, the control strategy of base station replacement is explained.

\subsection{Control Strategy}

Since the operation of the base station in the emergency situation like the navigation warfare situation assumes that the existing fixed base station can not be used, the additional arrangement of the mobile station is considered. In this case, the number of available mobile station can be extended to $\mathrm{N}$, and in the case where the fixed reference station arranged for the position estimation is available, the existing fixed reference station and the mobile reference station can be arranged together. When additional mobile reference stations were used, the estimation accuracy of DOA by measurement was analyzed on a Cramer-Rao lower bound (CRLB) basis.

The Cramer-Rao inequality lower bounds the covariance achievable by an unbiased estimator under two mild regularity conditions. Considering the unbiased estimate $\hat{p}$ for $p$, the Cramer-Rao bound states that

$$
E\left[(\hat{p}-p)(\hat{p}-p)^{T}\right] \geq I^{-1}(p)=C(p)
$$

where $I$ is the Fisher information matrix. In general, if $I$ is singular then no unbiased estimator for $p$ exists with a finite variance.

\subsection{DOA Based Localization and Placement}

The measured value of angle $\left(\theta_{i}\right)$ shown in Figure 1 is given by

$$
\hat{\theta}_{i}=\theta_{i}(p)+n_{i}=\arctan \left(\frac{y_{p}-y_{s i}}{x_{p}-x_{s i}}\right)+n_{i}
$$

where the arctan is defined such that $\theta_{i}(p) \in[0,2 \pi)$ and the $n_{i}$ is the measurement error. This error is assumed to be white Gaussian distribution with zero mean and variance $\sigma_{\theta}^{2}$, i.e., $n_{i} \sim N\left(0, \sigma_{\theta}^{2}\right)$.

Fisher information matrix $\left(I_{\theta}(p)\right)$ for $N$ number of base station around the target can be written as [3],

$$
I_{\theta}(p)=\frac{1}{\sigma_{\theta}^{2}} \sum_{i=1}^{N} \frac{1}{r_{i}^{2}}\left[\begin{array}{cc}
\sin ^{2} \theta_{i} & -\sin \theta_{i} \cos \theta_{i} \\
-\sin \theta_{i} \cos \theta_{i} & \cos ^{2} \theta_{i}
\end{array}\right]
$$

Especially, the Fisher information determinant for bearing-only localization can be given as,

$$
\operatorname{det}\left(I_{\theta}(p)\right)=\frac{1}{\sigma_{\theta}^{4}} \sum_{S} \frac{\sin ^{2}\left(\theta_{j}-\theta_{i}\right)}{r_{j}^{2} r_{i}^{2}}
$$

and

$$
\begin{aligned}
& \operatorname{det}\left(I_{\theta}(p)\right) \\
& =\frac{1}{4 \sigma_{\theta}^{4}}\left[\left(\sum_{i=1}^{N} \frac{1}{r_{i}^{2}}\right)-\left(\sum_{i=1}^{N} \frac{\cos 2 \theta_{i}}{r_{i}^{2}}\right)^{2}-\left(\sum_{i=1}^{N} \frac{\cos 2 \theta_{i}}{r_{i}^{2}}\right)^{2}\right]
\end{aligned}
$$

where $S=\{\{i, j\}\}$ is defined as the set of all combinations of $i$ and $j$ with $i, j \in\{1, \ldots, N\}$ and $j>i$, implying $|S|=\left(\begin{array}{c}N \\ 2\end{array}\right)$. Here $|\bullet|$ indicates the number of combinations.

In Figure 1, the location of GNSS interference source is at $P\left(x_{p}, \mathrm{y}_{p}\right)$ which is $b$ distance away from the $\mathrm{x}$-axis. $N$ distance number of AOA sensors (one fixed at the origin, $S_{1}$ ) are the $\mathrm{x}$-axis, separated by $\mathrm{x}$ distance from each other.

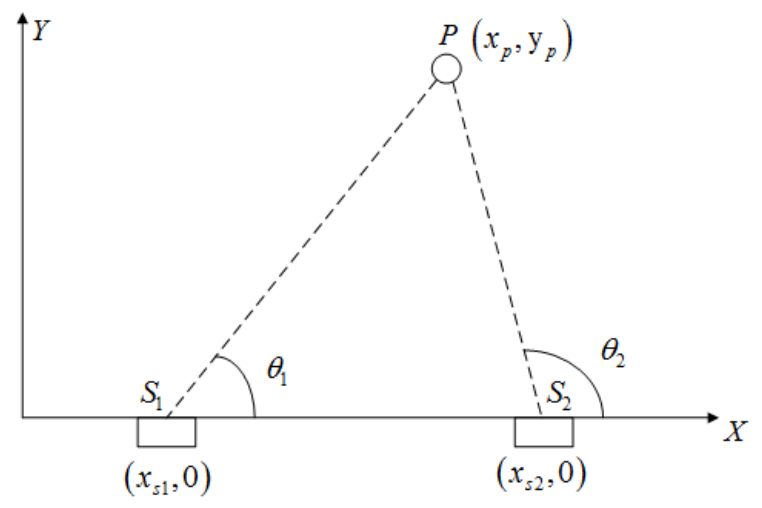

Fig. 1. Localization with two sensors (AOA) on x-axis. 
The Fisher information determination for this case is

$$
\begin{aligned}
& \operatorname{det}\left(I_{\theta}(p)\right) \\
& =\frac{1}{\sigma_{\theta}^{4}} \sum_{j=2}^{N} \sum_{i=0}^{j-2}\left\{\frac{b[j-(i+1)] x}{\left[(a-i x)^{2}+b^{2}\right]\left[(a-[j-1] x)^{2}+b^{2}\right]}\right\}^{2}
\end{aligned}
$$

where $(a, b)=\left(x_{p}, y_{p}\right)$. Translating Equation (4) into Cartesian coordinates and rearranging leads to Equation (6).

Consider that the target location is $P\left(x_{p}, \mathrm{y}_{p}\right)$ and the position of the fixed sensor $\left(S_{1}\right)$ and the line on which the second sensor to be placed is known as shown in Figure 1. The Fisher information determinant for this case is

$$
\operatorname{det}\left(I_{x}(p)\right)=\frac{1}{\sigma_{\theta}^{4}}\left(\frac{-y_{p} x_{s 2}}{\left(x_{p}^{2}+y_{p}^{2}\right)\left[\left(x_{p}-x_{s 2}\right)^{2}+y_{p}^{2}\right]}\right)^{2}
$$

By maximizing Equation (7) with respect to $x_{s 2}$, it can be shown that, $\operatorname{det}\left(I_{x}(p)\right)$ maximizes when,

$$
x_{s 2}= \pm \sqrt{x_{p}^{2}+y_{p}^{2}}
$$

Then the optimal distance between these two sensors is equal to the distance between the fixed sensor and the target $\left(\left\|S_{1}-S_{2}\right\|=\left\|S_{1}-P\right\|\right)$.

By using results, the additional rearranged mobile station follows the triangular layout with target's location (GNSS interference source) and the available fixed base station.

\section{Information-weighted Consensus Filter (ICF)}

Due to high fault-tolerance and scalability to large networks in the GNSS quality monitoring system in the navigation warfare situation, consensus-based distributed algorithms are needed. One of the recent consensus algorithms is the information-weighted consensus filter which deals with the system with unknown cross correlation term in the covariance of state variables. The ICF is guaranteed to converge to the optimal centralized performance under certain reasonable conditions. In addition, the ICF also supports multiple consensus iterations at each time step to improve performance compared to other methods.

In general, the state variables' and measurement model are expressed by [4]

$$
\mathbf{x}(t+1)=\mathbf{F} \mathbf{x}(t)+w(t)
$$

$$
\mathbf{z}_{i}(t)=\mathbf{H}_{i}(t) \mathbf{x}(t)+v_{i}(t)
$$

where the system noise $w(t)$ and the measurement noise $v_{i}(t)$ are simplify modelled as white Gaussian noise with zero mean and variance $\mathbf{Q}$ and $\mathbf{R}_{i}$, respectively.

After obtaining the measurement $\mathbf{z}_{i}$ and the measurement information matrix $\mathbf{B}_{i}=\mathbf{R}_{i}^{-1}$ at each sensor $i$, the initial information matrix $\mathbf{V}_{i}^{0}$ and the initial matrix vector $\mathbf{v}_{i}^{0}$ are calculated as shown in (11) and (12), respectively.

$$
\begin{aligned}
& \mathbf{V}_{i}^{0}=\frac{1}{N} \mathbf{W}_{i}^{-}(t)+\mathbf{H}_{i}^{T} \mathbf{B}_{i} \mathbf{H}_{i} \\
& \mathbf{v}_{i}^{0}=\frac{1}{N} \mathbf{W}_{i}^{-}(t) \mathbf{x}_{i}^{-}(t)+\mathbf{H}_{i}^{T} \mathbf{B}_{i} \mathbf{z}_{i}
\end{aligned}
$$

where $N$ presents the number of sensors, $\mathbf{x}_{i}^{-}(t)$ represents the prior state estimate, and $\mathbf{W}_{i}^{-}(t)$ refers to the prior information matrix which can be calculated by the reciprocal of the covariance of the prior state estimate. The information matrix $\mathbf{V}_{i}$ and the matrix vector $\mathbf{v}_{i}$ of $i$-th node sensor independently perform an average consensus, repeating (13) and (14) from $k=1$ to $k=K$ (consensus iteration).

$$
\begin{aligned}
& \mathbf{V}_{i}^{k}=\mathbf{V}_{i}^{k-1}+\varepsilon \sum_{j \in N_{i}}\left(\mathbf{V}_{j}^{k-1}-\mathbf{V}_{i}^{k-1}\right) \\
& \mathbf{v}_{i}^{k}=\mathbf{v}_{i}^{k-1}+\varepsilon \sum_{j \in N_{i}}\left(\mathbf{v}_{j}^{k-1}-\mathbf{v}_{i}^{k-1}\right)
\end{aligned}
$$

where $j$ means all neighboring sensors which exchange information with connected sensors as in (13) and (14). $\varepsilon$ is the rate parameter which should be chosen between 0 and $1 / \Delta \max$, where $\Delta \max$ is the maximum number of connected sensors. The rest of detail explanation on the ICF is written in $[4,5]$.

The process of the ICF is given below.

Inputs: prior state estimate $\mathbf{x}^{-}(t)$, prior information matrix $\mathbf{W}^{-}(t)$, observation matrix $\mathbf{H}_{i}$, measurement $\mathbf{z}_{i}$, measurement information matrix $\mathbf{B}_{i}$, consensus rate parameter $\varepsilon$, state transition matrix $\mathbf{F}$, process covariance $\mathbf{Q}$.

Compute initial information matrix and vector

$$
\begin{aligned}
& \mathbf{V}^{0}=\frac{1}{N} \mathbf{W}^{-}(t)+\mathbf{H}_{i}^{T} \mathbf{B}_{i} \mathbf{H}_{i}, \\
& \mathbf{v}^{0}=\frac{1}{N} \mathbf{W}^{-}(t) \mathbf{x}^{-}(t)+\mathbf{H}_{i}^{T} \mathbf{B}_{i} \mathbf{z}_{i}
\end{aligned}
$$

Perform average consensus on $\mathbf{V}^{0}$ and $\mathbf{v}^{0}$ independently 
for $k=1$ to $K$ do

$$
\begin{aligned}
& \mathbf{V}^{k}=\mathbf{V}^{k-1}+\varepsilon \sum_{j=1}^{N}\left(\mathbf{V}_{j}^{k-1}-\mathbf{V}^{k-1}\right), \\
& \mathbf{v}^{k}=\mathbf{v}^{k-1}+\varepsilon \sum_{j=1}^{N}\left(\mathbf{v}_{j}^{k-1}-\mathbf{v}^{k-1}\right)
\end{aligned}
$$

end

Compute a posteriori state estimate and information matrix for time $t$

$$
\mathbf{x}^{+}(t)=\left(\mathbf{V}^{k}\right)^{-1} \mathbf{v}^{k}, \mathbf{W}^{+}(t)=N \mathbf{V}^{k}
$$

Predict a state estimate and information matrix for time $(t+1)$

$$
\begin{aligned}
& \mathbf{x}^{-}(t+1)=\mathbf{F} \mathbf{x}^{+}(t), \\
& \mathbf{W}^{-}(t+1)=\left(\mathbf{F}\left(\mathbf{W}^{+}(t)\right)^{-1} \mathbf{F}^{T}+\mathbf{Q}\right)^{-1}
\end{aligned}
$$

Output: state estimate $\mathbf{x}^{+}(t)$, information matrix $\mathbf{W}^{+}(t)$

where $\mathbf{F}=\mathbf{I}, \mathbf{W}^{-}(t)$ can be calculated by the reciprocal of the covariance of the prior state estimate $\mathbf{x}^{-}(t)$, and $N$ presents the number of fusion information.

\section{Simulation Analysis}

In this section, in order to verify the optimal replacement method explained in the section 2 and localization performance of the proposed algorithm compared to conventional method, simulation is performed and analyzed.

\subsection{Simulation Conditions and Assumptions}

The simulation conditions are as follows. First, it is assumed that there is no fully network connection between the sub base stations and the mobile stations (12, 1-3 connected). Observations are direction angles of the received signal and the connected fixed station is limited to less than two stations every time for additional placement of mobile stations. The measured field of view (FOV) of each station was set to $200 \mathrm{~m}$ width and $200 \mathrm{~m}$ height and the direction angle estimation error was set to 4 degrees. For the comparative analysis of the position estimation performance, the information-weighted consensus filter (ICF), the Kalman consensus filter (KCF) [6] and the generalized KCF (GKCF) [7] were selected.

\subsection{Simulation Results}

Figure 3 shows the results of the position estimation using each method, and Figure 4 indicates the filtering errors of the $\mathrm{x}$-axis and $\mathrm{y}$-axis, respectively. Table 1 presents the comparison on the position estimation error expressed by root mean square error (RMSE) and the computation time of the algorithm (relative time for one sequence), using each method.

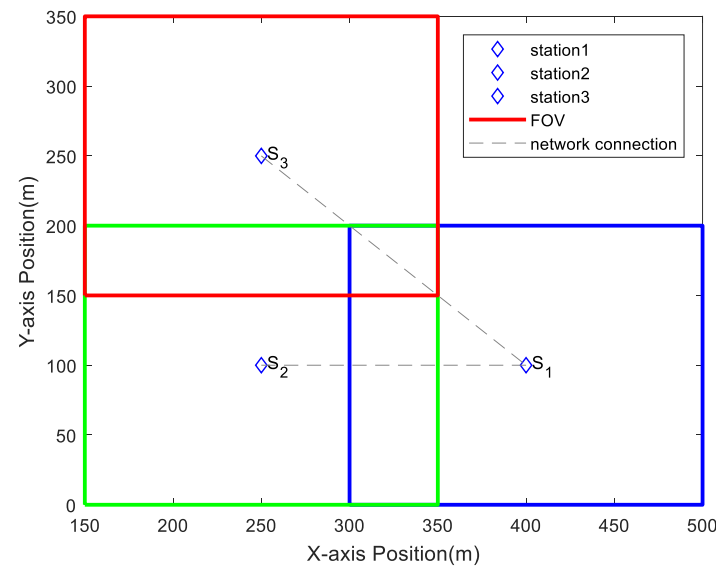

Fig. 2. Base station network and FOV.

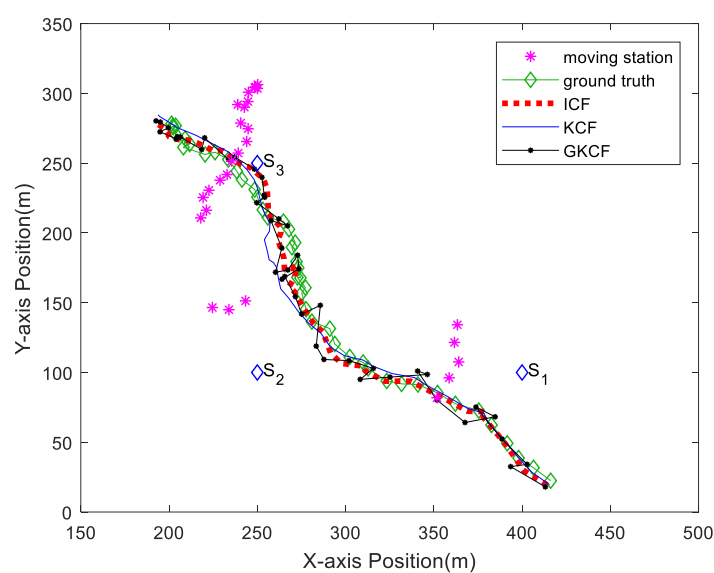

Fig. 3. Localization result.
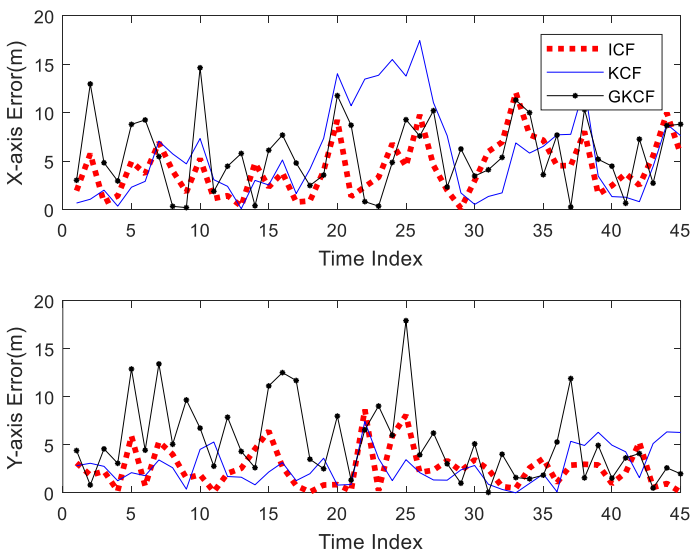

Fig. 4. Filtering error. 
Table 1. Estimation error and computation time.

\begin{tabular}{|c|c|c|}
\hline & RMSE(m) & Time(sec) \\
\hline ICF & 5.3735 & 0.0036 \\
\hline KCF & 6.6564 & 0.0032 \\
\hline GKCF & 8.8875 & 0.0038 \\
\hline
\end{tabular}

From the simulation results, it is confirmed that the estimation performance of the ICF, which uses the information of each measurement as weight, is better than that of the GKCF and KCF. The computation time difference of each technique was small although the ICF performed consensus iteration $(\mathrm{K}=20)$. If the quality of information fusion is not properly reflected as in the case of the GKCF, the performance is worse than the KCF. Also, it is confirmed that the position estimation performance can be improved by adding a mobile station in the case of the base stations are not fully connected as in the navigation warfare conditions.

\section{Conclusion}

The proposed algorithm deals with the optimization of the mobile station replacement with the fixed station for single target tracking effectively. In addition, this optimal placement method is applied to the ICF based localization method for tracking moving GNSS interference source. According to analysis results and simulation results, it is confirmed that triangular placement is the best arrangement type of mobile station and the proposed localization method has better performance compared to conventional methods in the distributed network situation which reflect the navigation warfare conditions.

This work has been supported by the National GNSS Research Center program of the Defense Acquisition Program Administration and Agency for Defense Development.

\section{References}

1. S.Y. Kim, C.H. Kang, C.G. Park, ISGNSS 2015, (2015)

2. S.Y. Kim, C.H. Kang, C.G. Park, K.H. Lee, M.J. Yu, KIMST 2017 (2017). (in Korean)

3. Sanvidha C.K. Herath, Pubudu N. Pathirana, Sensors, 13 (2013)

4. A.T. Kamal, J.A. Farrell, A.K. Roy-Chowdhury, 2012 IEEE 51st Conf. Decision and Control, Maui, HI, USA, pp. 2732-2737 (2012)

5. A.T. Kamel, J.A. Farrell, A.K. Roy-Chowdhury, IEEE Trans. Automat. Cont., 58 (2013)

6. R. Olfati-Saber, Proceedings of the 48th IEEE Conference on Decision and Control and 28th
Chinese Control Conference (CDC/CCC 2009), (2009)

7. A.T. Kamal, C. Ding, B. Song, J.A. Farrell, A.K. Roy-Chowdhury, Proceedings of the 50th IEEE Conference on Decision and Control and European Control Conference (CDC/ECC 2011), (2011) 